\title{
The complex karyotype landscape in chronic lymphocytic leukemia allows the refinement of the risk of Richter syndrome transformation
}

Haematologica 2022

Volume 107(4):868-876

\section{Correspondence:}

LIVIO TRENTIN

livio.trentin@unipd.it

ANDREA VISENTIN

andrea.visentin@unipd.it

Received: January 5, 2021.

Accepted: May 21, 2021.

Pre-published: June 3, 2021.

https://doi.org/10.3324/haematol.2021.278304

(C)2022 Ferrata Storti Foundation

Material published in Haematologica is covered by copyright. All rights are reserved to the Ferrata Storti Foundation. Use of published material is allowed under the following terms and conditions:

https://creativecommons.org/licenses/by-nc/4.0/legalcode. Copies of published material are allowed for personal or internal use. Sharing published material for non-commercial purposes is subject to the following conditions:

https://creativecommons.org/licenses/by-nc/4.0/legalcode, sect. 3. Reproducing and sharing published material for commercial purposes is not allowed without permission in writing from the publisher.

\author{
Andrea Visentin, ${ }^{1,2}$ Laura Bonaldi, ${ }^{3}$ Gian Matteo Rigolin, ${ }^{4}$ \\ Francesca Romana Mauro, ${ }^{5}$ Annalisa Martines, ${ }^{3}$ Federica Frezzato, ${ }^{1,2}$ \\ Stefano Pravato, ${ }^{1,2}$ Leila Romano Gargarella, ${ }^{1,2}$ Maria Antonella Bardi, ${ }^{4}$ \\ Maurizio Cavallari4, Eleonora Volta, ${ }^{4}$ Francesco Cavazzini, ${ }^{4}$ Mauro Nanni, ${ }^{5}$ \\ Monica Facco, ${ }^{1,2}$ Francesco Piazza, ${ }^{1,2}$ Anna Guarini, ${ }^{5}$ Robin Foà, ${ }^{5}$ \\ Gianpietro Semenzato, ${ }^{1,2}$ Antonio Cuneo ${ }^{4}$ and Livio Trentin ${ }^{1,2}$
}

${ }^{1}$ Hematology and Clinical Immunology Unit, Department of Medicine, University of Padua, Padua; ${ }^{2}$ Veneto Institute of Molecular Medicine, Padua; ${ }^{3}$ Immunology and Molecular Oncology Unit, Veneto Institute of Oncology IOV-IRCSS, Padua; ${ }^{4}$ Hematology section, Department of Medical Sciences, Azienda Ospedaliera-Universitaria, Arcispedale S. Anna, University of Ferrara, Ferrara; ${ }^{5} \mathrm{Hematology}$ division, Department of Precision and Translational Medicine, "Sapienza" University, Rome, Italy

\section{ABSTRACT}

omplex karyotype (CK) at chronic lymphocytic leukemia (CLL) diagnosis is a negative biomarker of adverse outcome. Since the impact of CK and its subtypes, namely type-2 CK (CK with major structural abnormalities) or high-CK (CK with $\geq 5$ chromosome abnormalities), on the risk of developing Richter syndrome (RS) is unknown, we carried out a multicenter real-life retrospective study to test its prognostic impact. Among 540 CLL patients, 107 harbored a CK at CLL diagnosis, 78 were classified as CK2 and 52 as high-CK. Twenty-eight patients developed RS during a median follow-up of 6.7 years. At the time of CLL diagnosis, CK2 and high-CK were more common and predicted the highest risk of RS transformation, together with advanced Binet stage, unmutated (U)-IGHV, 11q-, and TP53 abnormalities. We integrated these variables into a hierarchical model: high-CK and/or CK2 patients showed a 10-year time to RS (TTRS) of 31\%; U-IGHV/11q/TP53 abnormalities/Binet stage B-C patients had a 10-year TTRS of $12 \%$; mutated (M)-IGHV without CK and TP53 disruption a 10-year TTRS of $3 \%(P<0.0001)$. We herein demonstrate that CK landscape at CLL diagnosis allows the risk of RS transformation to be refined and we recapitulated clinico-biological variables into a prognostic model.

\section{Introduction}

Chronic lymphocytic leukemia (CLL), the most common leukemia in western countries, is a remarkably heterogeneous disease, with some patients never requiring treatments and others with a highly aggressive and/or rapidly progressive clinical course. ${ }^{1,2}$ Richter syndrome (RS) is the transformation of CLL into an aggressive lymphoma, most commonly resembling diffuse large B-cell lymphoma (DLBCL) or Hodgkin lymphoma (HL) variants. ${ }^{3,4}$ It is characterized by fast growing lymphadenopathies, 18-fluorodeoxyglucose (FDG) positron emission tomography computerized tomography (PET-CT)-avid masses, B symptoms, worsening performance status and increased lactate dehydrogenase levels. ${ }^{5}$ It is a challenging task to distinguish RS from progressive CLL and it is even more difficult to study prognostic markers since the frequency of RS transformation affects $2 \%-10 \%$ of CLL patients. ${ }^{5}$

Several studies have proved that chromosome banding analysis is able to refine the prognostic stratification of CLL compared to fluorescence in situ hybridization (FISH) analysis. In fact, 22\%-36\% of CLL cases with 'normal' FISH carry a chromosomal aberration following stimulated karyotypic analyses. In particular, complex karyotype (CK), defined by the presence of at least three chromosome lesions 
in the same clone, is detectable in $14 \%-34 \%$ of CLL cases. ${ }^{6-9}$ The presence of a CK is both a negative prognostic and predictive biomarker associated with an adverse outcome $e^{6,10}$ and worse response to chemoimmunotherapy, ${ }^{7,11}$ as well as to novel agents, ${ }^{12,13}$ regardless of the CLLIPI score or IGHV mutational status. ${ }^{8}$ However, the CK itself is a heterogeneous quantitative and qualitative cytogenetic category that includes numerical (i.e., monosomies and trisomies) and structural abnormalities (i.e., balanced and unbalanced translocations, marker chromosomes, isochromosomes, deletions, insertions and additions). Recently, collaborative studies have demonstrated that among CK cases assessed at CLL diagnosis, those harboring five or more chromosome abnormalities (high-CK) ${ }^{14}$ or those with major structural abnormalities, also called type-2 CK (CK2), ${ }^{15,16}$ identify highly aggressive disease subsets with a poor outcome; the latter is also characterized by a peculiar mRNA expression profile. ${ }^{15,17}$ Indeed, most of the patients included in these retrospective studies were managed with chemoimmunotherapy. ${ }^{14-16}$ However, the presence of CK has rarely been associated to the development of $\mathrm{RS}^{18}$ and, to date, it is unknown whether CK subtypes, namely high-CK or CK2, could help to identify patients at a higher risk of developing an RS at CLL diagnosis.

In this multicenter retrospective study, we documented, for the first time, that the presence of a CK at CLL diagnosis is associated with an increased risk of developing an RS. In particular, patients with CK2 and high-CK had the highest likelihood of RS transformation. Finally, we recapitulated clinico-biological variables associated with RS into a prognostic model defining three statistically distinct classes of risk of developing RS, the lowest risk for IGHV gene mutated (M-IGHV) being patients without any CK subtypes and an absence of TP53 abnormalities, and the highest risk for patients harboring highCK and/or CK2 subtypes.

\section{Methods}

\section{Study design}

Inclusion criteria for this study were diagnosis of CLL according to the 2008 iwCLL guidelines, ${ }^{19}$ histologically confirmed diagnosis of RS (diffuse large B-cell lymphoma or high-grade B-cell lymphoma), age $>18$ years and chromosome banding analysis performed within one year of diagnosis in patients without features of disease progression. Data included in the comparative analysis were gender, age, Binet stage, ${ }^{19} \mathrm{CLL}$ treatment prior to RS, 11q2223 deletion by FISH,$^{20}$ IGHV gene mutational analysis 21 and TP53 abnormalities including gene deletions (deletion 17p13) or mutations, ${ }^{22}$ and $\beta 2$-microglobulin level $>3.5 \mathrm{mg} / \mathrm{L}$. The primary endpoint was the impact of overall CK, CK2 and highCK on the time to Richter syndrome (TTRS) transformation. The correlation of RS with clinical and biologic variables and their impact on TTRS were secondary endpoints. This study was approved by the local research ethics committee and informed consent was obtained from all patients.

\section{Chromosome banding analysis}

Cytogenetic analysis was performed on peripheral blood after a 72h exposure to $500 \mu \mathrm{M} \mathrm{CpG} \mathrm{ODN} \mathrm{DSP30} \mathrm{(Roche,} \mathrm{Risch,} \mathrm{CH)}$ mitogen $+20 \mathrm{U} / \mathrm{mL}$ IL2 (Roche). Cultures were exposed overnight to $0.1 \mu \mathrm{g} / \mathrm{mL}$ colcemid $\left(\mathrm{Gibco}^{\circledR}\right.$ Karyomax Colcemid, ThermoFisher, Waltham, MA, USA) to obtain metaphases and then harvested following standard procedures. Karyotype was described after the analysis of at least 20 G-banded metaphases using IKAROS software (MetasYstems, Altlhusseim, Germany), according to international guidelines (ISCN 2016). The definition of a complex karyotype (CK) was defined by the presence of three or more chromosome abnormalities in the same clone. ${ }^{6,8,23,24}$ According to the literature, CK2 is represented by CK cases with major structural rearrangements that are unbalanced translocations, chromosome additions, insertion, duplications, ring, dicentric and marker chromosomes, whereas complex karyotypes with balanced translocations, deletions, monosomies or trisomies is defined as type-1 (CK1). ${ }^{16}$ High-CK cases were those presenting at least five chromosome abnormalities. ${ }^{14}$ Chromosome abnormalities found in only one metaphase were not considered as clonal, and were excluded. Karyotypes were reported by local cytogeneticists (AM, MAB and $M N$ ) and reviewed by $L B$ and $A V$.

Detail descriptions of IGHV mutational status, ${ }^{25-29}$ an assessment of stereotyped B-cell receptor (BCR), ${ }^{30,31}$ cytogenetics by fluorescence in situ hybridization (FISH), ${ }^{26,32}$ TP53 gene mutation, ${ }^{22}$ and NOTCH1 c.7544_7545delCT analysis ${ }^{33}$ are available in the Online Supplementary Methods.

\section{Statistical analysis}

Categorical variables were compared by the $\chi^{2}$ test (for Binet stages and FISH) or the Fisher exact test (gender, treatment, TP53 and $I G H V$ ), when appropriate. Continuous variables (median age) were compared using the Mann-Whitney test. TTRS was calculated starting from the date of CLL diagnosis to RS transformation (event) or last known follow-up (censored). ${ }^{19,34}$ Overall Survival (OS) was calculated starting from the date of CLL or RS diagnosis, when specified, to death for any cause, or to last known followup. Survival analyses were performed by the Kaplan-Meier method and the Log-rank test was used to compare survival curves between groups. The Cox regression model was employed to estimate hazard ratios (HR). The Cox proportional hazard assumption was assessed based on the scaled Schoenfeld residuals. The stability of our model was internally validated by the bootstrap 0.632 method with $\mathrm{B}=540$. The Harrell concordance index (c-index; 1.0 indicates a perfect discrimination, while a value of 0.5 indicates equivalence to chance) was used to compare our prognostic model. ${ }^{35}$ The prediction error was calculated as 1 - cindex, corrected for optimism and estimated using the 0.632 bootstrap method ${ }^{36}$ Akaike information criterium (AIC) was calculated using the AIC function with $\mathrm{R}$ (an open-source statistical package downloadable from $h t t p: / / w w w . r-p r o j e c t . o r g){ }^{37}$ A $P$ value $>0.05$ was considered as not significant.

\section{Results}

\section{Patients' characteristics}

We gathered data from 540 treatment-naive CLL patients with chromosome banding analysis assessed within 12 months from diagnosis at three Italian centers (Table 1). The median age at diagnosis of the whole case series was $63 \pm 12$ years, $61 \%$ were male, $75 \%$ showed Binet A stage, the median $\beta 2$-microglobulin was 2.93 $\mathrm{mg} / \mathrm{L}, 57 \%$ of patients were U-IGHV, $11 \%$ harbored TP53 abnormalities ( $8 \% 17 \mathrm{p} 13$ deletion and $3 \%$ only TP53 mutation) and $20 \%$ a CK (Figure S1A). NOTCH1 mutation was assessed in 47 patients at CLL diagnosis and it was found in two subjects who further developed RS. Two hundred and fifty-two patients subsequently received at least one line of therapy - 31\% FCR (fludarabine, cyclophosphamide, rituximab), 17\% BR (bendamus- 
Table 1. Clinical and biological features of patients.

\begin{tabular}{|c|c|c|c|c|}
\hline & $\begin{array}{l}\text { Population } \\
(n=540)\end{array}$ & $\begin{array}{c}\text { RS } \\
(n=28)\end{array}$ & $\begin{array}{c}\text { no RS } \\
(n=512)\end{array}$ & $\mathbf{P}$ \\
\hline \multicolumn{5}{|l|}{ Age (years) } \\
\hline median \pm sd & $63 \pm 12$ & $63 \pm 9.8$ & $63 \pm 12$ & 0.8793 \\
\hline \multicolumn{5}{|l|}{ Age at RS (years) } \\
\hline median \pm sd & $68 \pm 12$ & $68 \pm 12$ & - & n.a. \\
\hline \multicolumn{5}{|l|}{ Gender } \\
\hline Female & $211(39 \%)$ & $11(39 \%)$ & $200(39 \%)$ & $>0.9999$ \\
\hline Male & $329(61 \%)$ & $17(61 \%)$ & $312(61 \%)$ & \\
\hline \multicolumn{5}{|l|}{ Binet stage } \\
\hline A & $407(75 \%)$ & $15(54 \%)$ & $392(77 \%)$ & 0.0113 \\
\hline B - C & $133(25 \%)$ & $13(46 \%)$ & $120(23 \%)$ & \\
\hline \multicolumn{5}{|c|}{$\beta 2$-microglobulin $(\mathrm{mg} / \mathrm{L})^{*}$} \\
\hline median \pm sd & $2.9 \pm 1.5$ & $3.2 \pm 0.98$ & $2.9 \pm 1.6$ & 0.1216 \\
\hline \multicolumn{5}{|l|}{ IGHV status } \\
\hline M-IGHV & $232(43 \%)$ & $6(21 \%)$ & $225(44 \%)$ & 0.019 \\
\hline U-IGHV & $309(57 \%)$ & $22(79 \%)$ & $287(56 \%)$ & \\
\hline \multicolumn{5}{|l|}{ FISH } \\
\hline 13q or Normal & $404(75 \%)$ & $19(68 \%)$ & $385(75 \%)$ & 0.0861 \\
\hline+12 & $84(15 \%)$ & $3(11 \%)$ & $81(16 \%)$ & $0.0415^{+}$ \\
\hline $11 q-$ & $52(10 \%)$ & $6(21 \%)$ & $46(9 \%)$ & \\
\hline \multicolumn{5}{|l|}{ TP53 abn } \\
\hline Normal & $482(89 \%)$ & $19(68 \%)$ & $463(90 \%)$ & 0.0043 \\
\hline Disrupted & $58(11 \%)$ & $9(32 \%)$ & $49(10 \%)$ & \\
\hline \multicolumn{5}{|l|}{ KARYOTYPE } \\
\hline no CK & $433(80 \%)$ & $14(50 \%)$ & $419(82 \%)$ & 0.0002 \\
\hline CK & $107(20 \%)$ & $14(50 \%)$ & 93 (18\%) & \\
\hline \multicolumn{5}{|l|}{ QUALITATIVE } \\
\hline no CK & $433(80 \%)$ & $14(50 \%)$ & $419(82 \%)$ & $<0.0001$ \\
\hline CK1 & $29(5 \%)$ & $1(4 \%)$ & $28(5 \%)$ & \\
\hline CK2 & 78 (14\%) & $13(46 \%)$ & $65(13 \%)$ & \\
\hline \multicolumn{5}{|l|}{ QUANTITATIVE } \\
\hline 0 & $165(30 \%)$ & $3(11 \%)$ & $162(32 \%)$ & $<0.0001$ \\
\hline $1-2$ & 269 (50\%) & $11(39 \%)$ & $258(50 \%)$ & \\
\hline $3-4$ & $54(10 \%)$ & $3(11 \%)$ & $52(10 \%)$ & \\
\hline$\geq 5$ & $52(10 \%)$ & $11(39 \%)$ & $41(8 \%)$ & \\
\hline \multicolumn{5}{|l|}{ RS SCORE } \\
\hline low-risk & $212(39 \%)$ & $3(11 \%)$ & $209(41 \%)$ & $<0.0001$ \\
\hline Int.-risk & $247(46 \%)$ & $12(43 \%)$ & $235(46 \%)$ & \\
\hline high-risk & $81(15 \%)$ & $13(46 \%)$ & $68(13 \%)$ & \\
\hline
\end{tabular}

RS: Richter syndrome; sd: standard deviation; M-IGHV: mutated IGHV gene; U-IGHV: unmutated IGHV gene; 11q-: del11q22-23 by interphase FISH analysis; TP53 abn:TP53 abnormalities include deletions and/or mutations; CK: complex karyotype; CK1: type-1 CK; CK2: type-2 CK; highCK: $\geq 5$ chromosome abnormalities; high-risk: CK2 and/or highCK; int.risk: U-IGHV/11q-/TP53abn/Binet B-C; low-risk: M-IGHV without CK and TP53 wild type; n.a.: not applicable; * data available from 520 (96\%) patients, 26 (93\%) who developed an RS and 494 (96\%) who did not transform; + Analysis between subgroups with 11q- and others.

tine, rituximab), $10 \%$ ibrutinib, $5 \%$ chlorambucil plus an anti-CD20 monoclonal antibody, $2 \%$ venetoclax, $35 \%$ other treatments such as FC or chlorambucil single agent as first line therapy - and 90 died during the follow-up.

According to the qualitative CK subtype, 29 of 107 (27\%) patients displayed a CK1 and $78(73 \%)$ a CK2 (Online Supplementary Figure S1A, Table 1) whereas, according to the number of chromosome lesions, 165 $(30 \%)$ patients had a normal karyotype (i.e., $46,(\mathrm{XX})$ or 46,(XY) for females and males, respectively), 268 (50\%) had one or two lesions, and 54 (10\%) three or four abnor- malities and $52(10 \%)$ were classified as high-CK (i.e., $\geq 5$ chromosome lesions) (Online Supplementary Figure S1A and $S 2 A$, Table 1). In particular, a high-CK was more common in CK2 than in CK1 patients, being present in $63 \%$ of patients harboring a CK2 subtype but in only $10 \%$ of CK1 patients $(P<0.0001$, Online Supplementary Figure S2A).

As a preliminary step for our further analysis, we confirmed the established prognostic role of overall CK, CK with major unbalanced abnormalities (i.e., CK2) and highCK in our dataset (Figure S2B-D). The 10-year OS was $54 \%$ and $79 \%$ for CK and no-CK patients, respective- 
Table 2. Hazard ratios (HR) for the time to Richter syndrome.

\begin{tabular}{|c|c|c|c|c|c|c|}
\hline & & ariate ana & & & ivariate an & \\
\hline & HR & 95\% C.I. & $P$ & HR & $95 \%$ C.I. & $P$ \\
\hline TTRS & & & & & & \\
\hline$\geq 65$ years & 1.4 & $0.6-2.9$ & 0.4289 & - & - & - \\
\hline Age+ & 1.02 & $0.9-1.1$ & 0.2120 & - & - & - \\
\hline Male & 1.0 & $0.5-2.1$ & 0.9379 & - & - & - \\
\hline$\beta 2 \mathrm{MG}$ high* & 1.8 & $0.6-5.6$ & 0.2925 & - & - & - \\
\hline Binet B-C & 3.9 & $1.6-9.6$ & 0.0024 & 2.9 & $1.4-6.3$ & 0.0039 \\
\hline U-IGHV & 4.0 & $1.9-8.6$ & 0.0004 & 4.5 & $1.8-11.3$ & 0.0011 \\
\hline+12 & 0.8 & $0.3-2.4$ & 0.6675 & - & - & - \\
\hline $11 q-$ & 4.6 & $1.3-16.7$ & 0.0215 & 2.8 & $1.1-6.9$ & 0.0285 \\
\hline TP53 abn & 9.5 & 2.9-31.4 & 0.0002 & 3.9 & $1.8-8.7$ & 0.0008 \\
\hline $\mathrm{CK}$ & 7.4 & $3.0-18.3$ & $<0.0001$ & 4.7 & $2.2-9.9$ & $<0.0001$ \\
\hline CK2 & 8.8 & $4.9-19.8$ & $<0.0001$ & 5.6 & $2.7-11.8$ & $<0.0001$ \\
\hline High-CK & 9.9 & $6.5-22.9$ & $<0.0001$ & 6.9 & $3.3-14.9$ & $<0.0001$ \\
\hline RS MODEL & & & & & & \\
\hline Low-risk & 1.0 & - & - & 1.00 & - & - \\
\hline Int.-risk & 4.0 & $1.4-11.4$ & 0.0101 & 3.4 & $1.5-7.5$ & 0.0023 \\
\hline High-risk & 13.6 & 7.1-20.9 & $<0.0001$ & 9.2 & 4.7-17.3 & $<0.0001$ \\
\hline
\end{tabular}

TTRS: time to Richter syndrome; $\beta 2 \mathrm{MG}$ high: beta2-microglobulin $>3.5 \mathrm{mg} / \mathrm{L}$; U-IGHV: unmutated IGHV gene; TP53 abn: TP53 abnormalities include deletions and/or mutations CK: complex karyotype; CK1: type-1 CK; CK2: type-2 CK; highCK: $\geq 5$ chromosome abnormalities; High-risk: CK2 and/or highCK; Int-risk: U-IGHV/11q-/TP53abn/Binet B-C; Lowrisk: M-IGHV without CK and TP53 wild type; n.a.: not applicable; + : age considered as continuous variable; * : data available from 520 (96\%) patients, 26 (93\%) who developed an RS and 494 (96\%) who did not transform.

ly $(P<0.0001$, Online Supplementary Figure S2B); 48\% vs. $72 \%$ vs. $79 \%$ for CK2, CK1 and no-CK $(P<0.0001$, Online Supplementary Figure S2C), respectively; $44 \%$ vs. $64 \%$ vs. $70 \%$ vs. $90 \%$ for patients with $\geq 5$ (i.e., high-CK), 4-3, $2-1$ and without chromosome abnormalities $(P<0.0001$, Online Supplementary Figure S2D), respectively.

\section{Clinico-biological features of patients who developed a RS transformation}

Twenty-eight (5.2\%) patients developed a histologically confirmed RS over a median follow-up of 6.7 years (Figure $\mathrm{S} 1 \mathrm{~B})$. The median age at RS diagnosis was 68 years (range $38-84)$, $61 \%$ were male, $75 \%$ had received a CLL treatment in the past, $79 \%$ were U-IGHV, $32 \%$ presented TP53 abnormalities, $50 \%$ harbored a CK at CLL diagnosis, which included $46 \%$ and $39 \%$ of CK2 and high-CK subtypes, respectively. Eight cases showed deletion of 9p21.3, i.e., the locus of CDKN2A gene, all with a CK. In particular, 8 of 8 were classified as CK2 and 6 of 8 as high-CK subtype. Only one patient, who developed RS, received ibrutinib frontline. We also observed that more patients who developed an RS displayed an advanced Binet stage at CLL diagnosis $(P=0.0113)$ and were enriched in U-IGHV $(P=0.0191)$, TP53 abnormalities $(P=0.0043), C K$ overall $(P=0.0002)$, CK2 $(P<0.0001)$ and high-CK $(P<0.0001)$ cases as compared to patients who did not develop an RS (Table 1, Figure S1C). Age at CLL diagnosis (median age 63.5 and 63.3 years), gender distribution (both 61\%), trisomy of chromosome 12 (11\% and $16 \%), \beta 2$-microglobulin (median levels $3.2 \mathrm{mg} / \mathrm{L}$ and $2.9 \mathrm{mg} / \mathrm{L}$ ) and stereotyped BCR $(10.7 \%$ vs. $9.8 \%)$ had a superimposable distribution among patients with and without an RS transformation (Table 1).

\section{Prognosticators of Richter Syndrome}

The cumulative incidence of RS slowly increases over time. As shown in Figure 1A, 2.6\%, 12\% and 13\% of patients developed an RS within five, ten and 15 years after CLL diagnosis, respectively. We observed that patients with a CK, overall (Figure $1 \mathrm{~B}$ ) and its subtypes (Figure 1C-D), had a very high risk of developing an RS.

The estimated ten-year TTRS to be $25 \%$ vs. $8 \%$ $(P<0.0001), 38 \%$ vs. $8 \%(P<0.0001)$ and $41 \%$ vs. $8 \%$ $(P<0.0001)$ for patients with CK vs. no-CK, CK2 vs. other patients (i.e., CK1 or noCK), highCK vs. other patients (i.e., 3-4 or 1-2 or, 0 chromosome abnormalities) respectively (Figure 1C-D and S3G-H). Multivariate analysis revealed that $\mathrm{CK}$ overall was associated with a more than four-fold higher risk of developing an RS (HR 4.7, 95\% CI 2.2-9.9, $P<0.0001)$. This risk was even higher for CK subtypes, being more than five-fold (HR 5.6, 95\% CI 2.7-11.8, $P<0.0001)$ and seven-fold (HR 6.9, 95\% CI 3.3-14.9, $P<0.0001)$ higher for patients harboring CK2 and high-CK subtypes, respectively (Table 2 ). Other variables associated with TTRS at univariate and multivariate analysis were Binet stage B-C, U-IGHV, 11q-, TP53 abnormalities (Table 2, Online Supplementary Figure S3A-E).

Among CK2 and/or high-CK patients ( $\mathrm{n}=81), 32$ (39\%) patients carried TP53 abnormalities and $52(63 \%)$ an U-IGHV status. We found that TP53 abnormalities and IGHV status mildly impact on the risk of developing RS among CK2 and/or high-CK subgroup, but the difference was not statistically significant (Figure S4A-B, $P=0.1150$ and $P=0.1405$, respectively). These data suggest that $C K$ subtypes per se represent a stronger prognosticator of RS transformation than conventional biologic markers such as TP53 disruption and U-IGHV conformation.

The median OS from CLL diagnosis for the whole population was not reached and the estimated ten-year OS was $73 \%$ (Figure S5A). Patients who developed an RS had a shorter OS (Figure 2A). The median OS was seven years $v s$. not reached and the estimated ten-year OS was $16 \%$ 


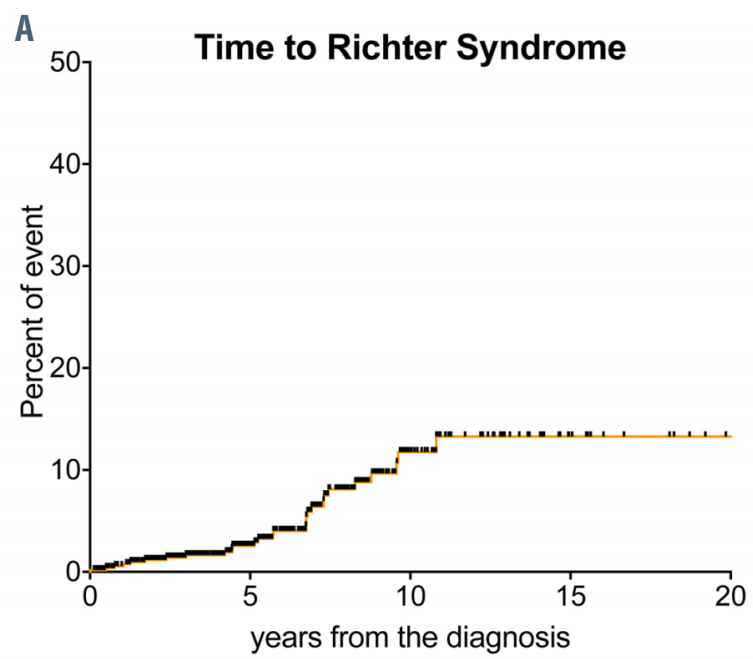

C

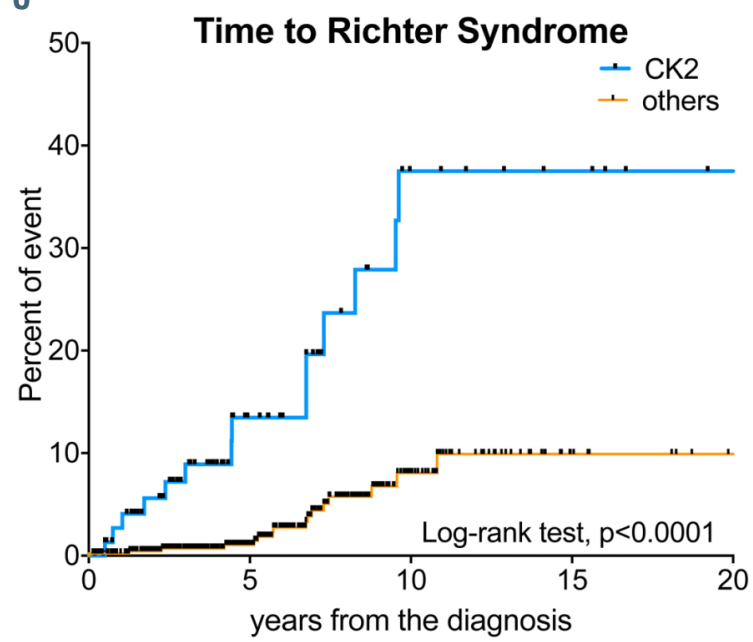

B

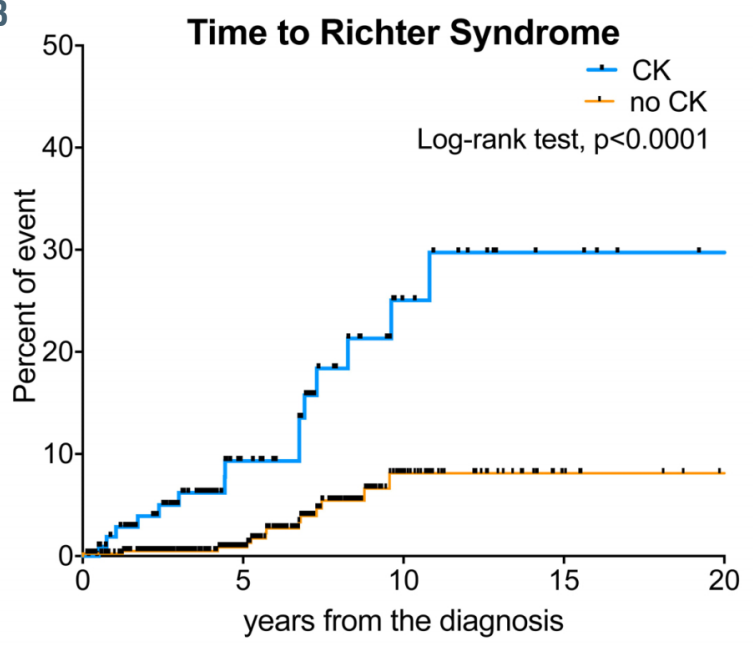

D

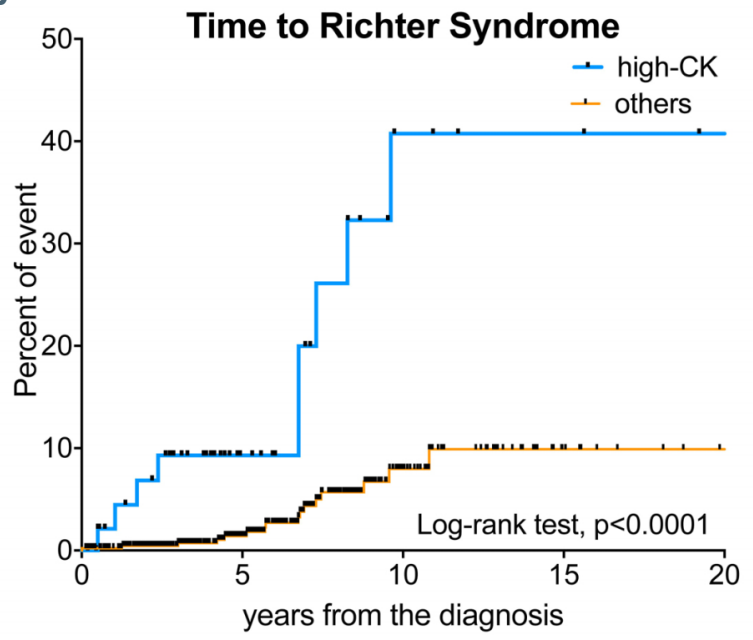

Figure 1. Kaplan Meyer curves of time to Richter syndrome. The upper-left (A) panel shows the time to Richter syndrome (RS) transformation for the whole population. Patients with a CK overall (B), CK2 (C) or high-CK (D) have a significantly increased a risk of developing an RS compared to the other patients (Log-rank test, $P<0.0001)$.

vs. $79 \%$ for patients who developed an RS vs. those who did not transform (Figure $2 \mathrm{E}, P<0.0001$ ), respectively. Variables that were associated with a higher risk of death in multivariate analysis are summarized in Table S1.

The median time from CLL diagnosis to RS transformation was 5.3 years. ranging from 0.10 years to 10.8 years. In only one patient was RS was diagnosed within six months of CLL diagnosis. The median OS from RS transformation was 5.3 months and the two-year OS was only $20 \%$ (Figure 2B). The OS from RS was not affected by the presence of a CK at CLL diagnosis nor its subtypes (Figure S5B-C). The two-year OS from RS was $28 \%$ vs. $10 \%$ for $\mathrm{CK} 2$ cases and other patients (i.e., CK1 and no-CK) $(P=0.3317)$ and $24 \%$ vs $16 \%$ for high-CK cases and other patients, respectively (i.e., $<5$ chromosome abnormalities) $(P=0.9864)$ (Figure S5C-D). No traditional prognostic markers could foresee the risk of death after RS diagnosis in our population (Table S2).

\section{A Richter syndrome prognostic model}

By integrating CK subtypes, TP53 abnormalities, 11q deletion, IGHV mutational status and Binet stages based on $\mathrm{HR}$ values, we developed a hierarchical model leading to the identification of three statistically different groups. These were ranked from the shortest to the longest TTRS, as follows: $81(15 \%)$ patients were classified as high-CK and/or CK2, and had a five-year and ten-year TTRS of $13 \%$ and $31 \%$; $247(46 \%)$ patients displayed a U-IGHV status or 11q- or TP53 disruption or Binet stage B-C, and showed the five-year and ten-year TTRS of $0.9 \%$ and $12 \% ; 212$ (39\%) patients were M-IGHV without CK and TP53 abnormalities, and had a five-year and ten-year TTRS of $0.7 \%$ and $3 \%$ (Figure $3, P<0.0001$ ). Multivariate analysis confirmed that the former subgroup (i.e., high-CK and/or CK2) was associated with the highest risk of RS transformation (HR 9.2, 95\% CI 3.8-46, $P<0.0001$ ) compared to the low-risk group, one which is characterized by the presence of M-IGHV without CK and TP53 abnormalities. Patients with U-IGHV or 11q- or TP53 abnormalities or Binet stage B-C had an intermediate risk, with a threefold higher risk of RS compared to low-risk patients (HR $3.4,95 \%$ CI 1.5-7.5, $P=0.0023$ ) (Table 2). Our model was also internally validated using the bootstrap 0.632 method showing a prediction error of 0.26 . Finally, the c-index for our proposed model was 0.81 for TTRS and the Akaike information criterium was 286 . These results indicate that 
A

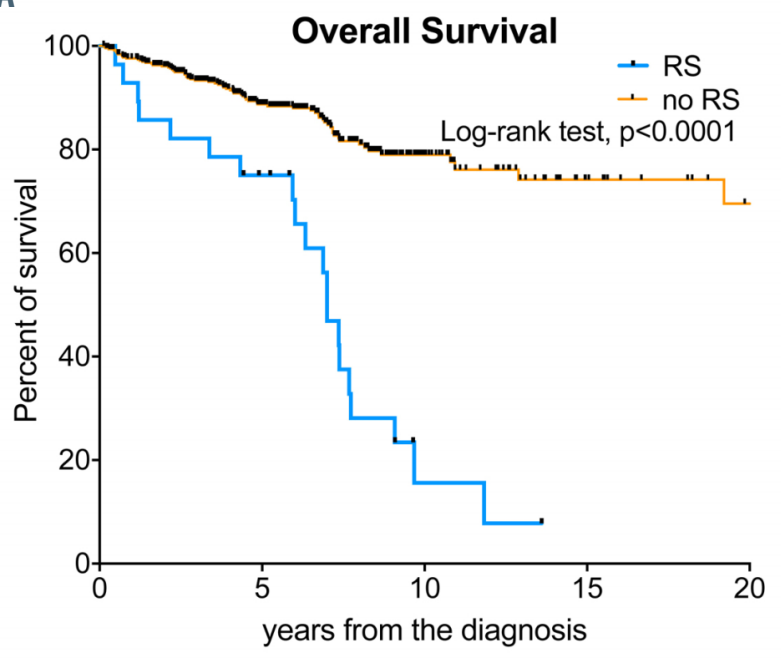

B

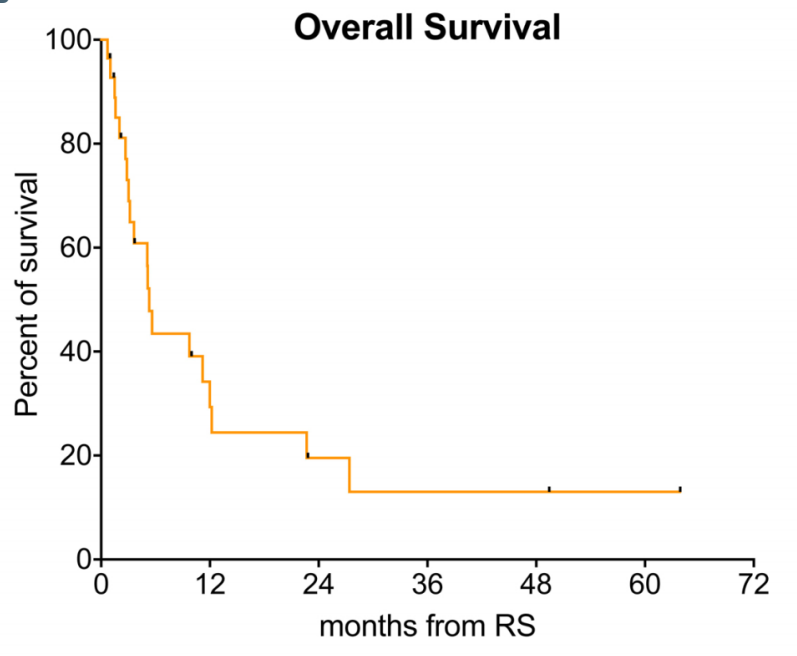

Figure 2. Kaplan Meyer curves of overall survival. The left panel (E) shows the overall survival analysis for patients with RS transformation and those who did not develop an RS (no RS). Patients who developed an RS had a shorter overall survival, calculated from CLL diagnosis (Log-rank test, $P<0.0001)$. The right (F) panel shows the overall survival after RS transformation, confirming these patients' very poor prognosis.

our model had a good prediction accuracy for the risk of developing an RS; higher than of the CLL-IP ${ }^{38}$ (c-index 0.69 , prediction error 0.28 , Akaike information criterium 301 ) and the Barcelona-Brno ${ }^{39}$ (c-index 0.74, prediction error 0.25 , Akaike information criterium 292) scores accuracies applied to our population (Figure S4C-D). Based on the lower Akaike score, our RS prognostic model better predicts the risk of developing an RS than the available comparators.

\section{Discussion}

In this multicenter retrospective study, we demonstrated that patients harboring a CK at CLL diagnosis, in particular those with $\mathrm{CK} 2$ and/or high-CK, are characterized by the highest risk of developing an RS transformation. Subsequently, by integrating data of CK subtypes with other clinical and biologic variables associated with the risk of RS, we were able to define an RS prognostic model. To minimize selection and attrition biases, as well as imprecise reporting of data inherent to observational studies, we asked the clinicians to report all patients who performed stimulated cytogenetic analysis within the first year of diagnosis. We analyzed the reported data and performed computerized and manual consistency checks on each case report form.

RS is a rare and an aggressive complication of CLL patients, affecting between $2 \%$ and $10 \%$ of CLLs. ${ }^{34}$ Most RS patients are elderly, have a poor performance status and suffer from several comorbidities which limit the use of intensive chemoimmunotherapy. ${ }^{40}$ Since the majority of patients are primary refractory to first-line treatment and only a few are able to undergo allogenic stem cell transplantation procedures, the reported estimated survival after a diagnosis of RS is usually less than one year, even with the introduction of targeted-therapy ${ }^{41,42}$ and immune checkpoint inhibitors. ${ }^{43}$ For these reasons, the standard of care of patients with RS remains a primary unmet need. Known biologic risk factors for the development of RS are TP53 and CDKN2A aberrations, NOTCH1 mutation and a stereotype BCR subset \#8. ${ }^{44,45}$ To date, the impact of CK at CLL diagnosis on the risk of developing RS has been investigated in only a few studies. ${ }^{46,47}$

The German CLL study group has recently reviewed the clinical features of RS patients as part of their clinical trials. ${ }^{34}$ In this study, $3.5 \%$ of CLL developed an RS transformation after a median observation time of 4.4 years. The median age at RS was 65 years and the median OS after RS was 9.4 months, which was significantly longer for HL compared to the DLBCL variant (median OS 83 months vs. 8.7 months, respectively). Adverse risk factors at trial enrollment, such as $17 \mathrm{p} 13$ deletion by FISH, high $\beta 2$-microglobulin and CLL-IPI scores were more common in patients who developed an RS34 while NOTCH1 mutations and stereotype \#8 were not recurrent in RS cases. ${ }^{34}$ Conversely, among the 204 RS from the Mayo clinic, the median OS after RS diagnosis was 12 months. ${ }^{48}$ In a multivariate Cox regression analysis, prior CLL treatment and older age, but not TP53 disruption, were associated with a shorter OS. ${ }^{48}$ The results of our real-life study are in line with the GCLLSG and Mayo clinic reports, even though our patients were slightly older; this could explain the shorter OS in our RS cohort (median survival after RS is 5.3 months). Comparable survival rates, between six and 12 months, have been observed in other retrospective analyses. ${ }^{34,44,48}$ In addition, advanced Binet stage, U-IGHV and 11q- were also significantly associated with an RS risk in our patients.

Chromosome banding analysis in CLL is capable of identifying chromosomal abnormalities that are missed by FISH analysis, sometimes fulfilling CK criteria. ${ }^{6,24,49,50}$ Genomic microarrays have also emerged as a valuable tool for genome-wide studies in CLL. However, in a recent study, no significant differences emerged in patients' classification, time to first treatment, OS and prediction accuracy between chromosome banding analysis and genomic microarrays. ${ }^{51}$ The prognostic and predictive role of CK, defined by the presence of at least three chromosomal lesions, is evident at diagnosis, ${ }^{6,8}$ as well as at disease progression $^{7}$ and in relapsed/refractory patients treated with ibrutinib ${ }^{13,52}$ or venetoclax. ${ }^{12}$ Of note, CK was not a prog- 


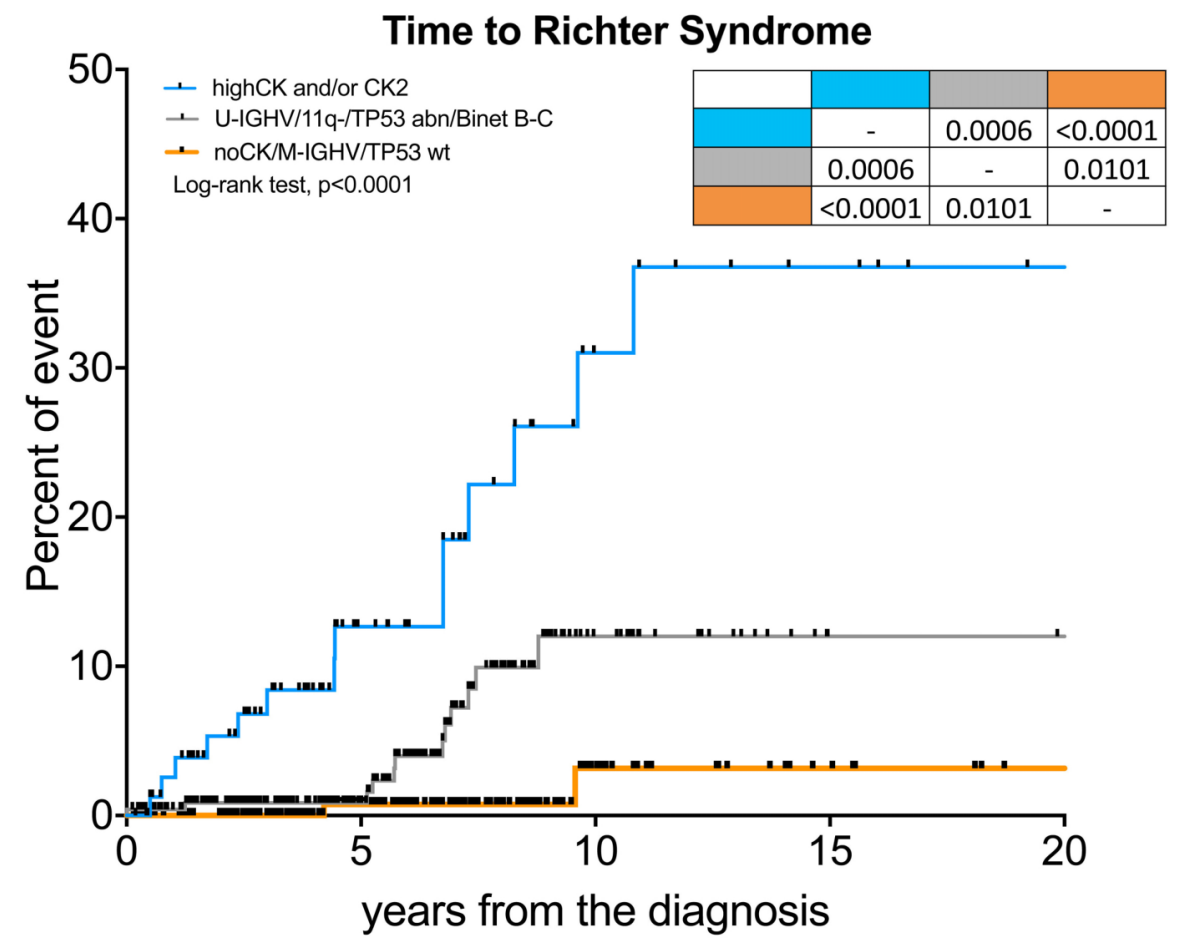

Figure 3. The Richter syndrome scoring system. Kaplan-Meier curve of time to Richter syndrome transformation according to the Richter syndrome scoring system. Patients were classified at high-risk if they were high-CK and/or CK2 at CLL diagnosis (blue curve); at intermediate-risk if they displayed unmutated IGHV status (UIGHV), 11q22-23 deletion (11q), TP53 abnormalities (including deletions or mutations, TP53 abn) or Binet stage B-C (grey curve); at low-risk if they were IGHV mutated (M-IGHV) patients without CK and wild-type TP53 gene (TP53 not deleted non mutated) (orange curve).

nostic marker of survival on multivariate analysis for patients treated frontline with ibrutinib \pm rituximab $b^{53}$ while treatment with idelalisib plus rituximab seems to have a comparable efficacy in $\mathrm{R} / \mathrm{R}$ patients with and without CK. ${ }^{54-56} \mathrm{CK}$ has been found in $14 \%-35 \%$ of CLL depending on the study in question, ${ }^{6,10}$ and identifies a heterogeneous cytogenetic category in terms of quantitative and qualitative characteristics. Data from the literature has documented that the presence of at least five chromosomal aberrations is associated with a very aggressive clinical course independent of the IGHV status and TP53 lesions. ${ }^{14}$ Our collaborative group has previously demonstrated that almost $70 \%$ of CK cases harbor major structural aberrations such as unbalanced translocations and ring or marker chromosomes. ${ }^{15}$ This subset, called CK2, was associated with a higher incidence of TP53 aberrations, chemo-refractoriness, early relapse after chemoimmunotherapy, and a shorter OS at multivariate analysis. ${ }^{15}$ In addition, the prognostic and predictive accuracy of CK subtypes is enhanced when it is combined with IGHV mutational status. ${ }^{16}$ Interestingly, a recent analysis of the international CLL ${ }^{14}$ clinical trial suggests that the fixedduration combination of obinutuzumab plus venetoclax seems to overcome the negative predictive impact of $\mathrm{CK}$, both in terms of undetectable minimal residual disease rates and progression-free survival. ${ }^{57}$

The presence of CK has been sporadically linked to the development of RS. ${ }^{18}$ In a retrospective study on CLL patients treated with FCR, one of four cases with RS had a CK. ${ }^{9}$ Anderson et al. ${ }^{12}$ found a CK in $48 \%$ of the 25 patients who progressed on venetoclax, including eight of 17 patients with RS. Rogers et al., ${ }^{47}$ reported a CK in $67 \%$ patients who developed an RS and found that a CK had an adverse impact on the R-EPOCH regimen. A recent study from Ohio State University found that six of nine patients with a near-tetraploidy (four copies of most chromosomes) karyotype developed an RS. ${ }^{46}$ At multivariate analysis, near-tetraploidy and CK predicted ibrutinib discontinuation due to transformation. ${ }^{46}$ Although the exact mechanism that favors the development of an RS in patients with CK is unknown, the strong association between CK and TP53 abnormalities, short telomere length and, consequently, increased chromosome instability could play a relevant role. . $^{8,58}$

Thanks to stimulated chromosome banding analysis, we were able to identify a CK in $20 \%$ of 540 CLL patients and could demonstrate that patients harboring a CK2 or a high-CK had a six and seven-fold increased risk of developing RS. We therefore suggest that the integration of CK subtypes, together with IGHV mutational status, TP53 abnormalities, 11q22-23 deletion and Binet stage, may allow the prognostic risk of RS transformation (Figure 3) to be refined. Indeed, we could show that M-IGHV patients without any CK subtypes and a wild-type TP53 gene are characterized by a very low risk of developing $\mathrm{RS}$, at only $0.7 \%$ five years from CLL diagnosis. On the other hand, patients with CK subtypes, both CK2 and/or high-CK, are characterized by the highest risk of developing RS, with $31 \%$ of them experiencing a disease transformation within ten years of diagnosis. In addition, our model seems to better predict the risk of RS transformation than the available scoring systems. Our results, like most data found in the literature, derived from a cohort of patients treated mainly with chemoimmunotherapy, a choice made partly due to their longer follow-up. Although the cumulative incidence of RS among patients 
treated with chemo/chemoimmunotherapy seems to be higher than patients treated with BTK or BCL2 inhibitors, this difference was not statistically significant $(P=0.3337$, Online Supplementary Figure S5E). In addition, after validation by an independent cohort of patients treated frontline with targeted drugs and in a prospective study, our prognostic model might be used in the follow-up management of patients with CLL. In particular, patients with a CK2 and/or high-CK should be carefully monitored for the development of an RS during their follow-up.

\section{Disclosures}

$A V$ received honoraria from Janssen, Abbvie, Italfarmaco. $L T$ received research funding by Gilead, Roche, Janssen and Takeda, advisory board for Roche, Shire and Abbvie, Astrazeneca. GMR received research funding by Gilead. FRM advisory board for Janssen, Shire and Abbvie. AC advisory board and speaker bureau for Roche, Abbvie, Gilead and Janssen. GS board member of Abbvie, Roche, Janssen and Celgene. RF advisory board or speaker bureau for Roche, Abbvie, Celgene, Incyte, Amgen, Janssen, Gilead and Novartis.

\section{Contributions}

$A V$ designed the study, performed statistical analysis, visited patients and wrote the article; SP, LRG, MC, EV and FC and provided intellectual inputs and visited patients; $L B, A M, M A B$ and $M N$ performed cytogenetic tests; $F F, M F$ and $A G$ performed cytofluorimetric and IGHV analysis; FRM, GMR, PF, GS, RF $A C$ and $L T$ visited patients, provided intellectual inputs and reviewed the article.

\section{Funding}

This work was supported by funds from Associazione Italiana per la Ricerca sul Cancro (A.I.R.C.) projects to LT (IG-25024), Gilead fellowship program 2018 to LT, Special Program 'Metastatic disease: the key unmet need in oncology', AIRC $5 \times 1000$ (No. 21198) to RF, Fondo di Ateneo per la Ricerca 2016, 2017 of the University of Ferrara to GMR and FC, Fondo di Incentivazione alla Ricerca 2017 of the University of Ferrara to GMR, Ministero dell'Istruzione, dell'Università e della Ricerca PRIN 2015 to AC (2015ZMRFEA). AV received a research fellowship from the University of Padua supported by ONLUS Ricerca per Credere nella Vita (RCV) odv, Padua, Italy.

This study was approved by the local research ethics committee of Padua hospital and informed consent was obtained from all patients.

\section{Data sharing statement}

The datasets generated and analyzed during the current study are not publicly available due to the data protection and lack of consent from the patients. Access to data is strictly limited to the researchers who have obtained permission for data processing.

\section{References}

1. Scarfo L, Ferreri AJ, Ghia P. Chronic lymphocytic leukaemia. Crit Rev Oncol Hematol. 2016;104:169-182.

2. Visentin A, Facco M, Frezzato F, et al. Integrated CLL scoring system, a new and simple index to predict time to treatment and overall survival in patients with chronic lymphocytic leukemia. Clin Lymphoma Myeloma Leuk. 2015;15(10):612-620

3. Mauro FR, Galieni P, Tedeschi A, et al. Factors predicting survival in chronic lymphocytic leukemia patients developing Richter syndrome transformation into Hodgkin lymphoma. Am J Hematol. 2017; 92(6):529-535.

4. Visentin A, Imbergamo S, Gurrieri C, et al. Major infections, secondary cancers and autoimmune diseases occur in different clinical subsets of chronic lymphocytic leukaemia patients. Eur J Cancer. 2017; 72:103-111.

5. Vitale C, Ferrajoli A. Richter syndrome in chronic lymphocytic leukemia. Curr hematol Malig Rep. 2016;11(1):43-51.

6. Baliakas P, Iskas M, Gardiner $A$, et al. Chromosomal translocations and karyotype complexity in chronic lymphocytic leukemia: a systematic reappraisal of classic cytogenetic data. Am J Hematol. 2014; 89(3):249-255

7. Herling CD, Klaumunzer M, Rocha CK, et al. Complex karyotypes and KRAS and POT1 mutations impact outcome in CLL after chlorambucil-based chemotherapy or chemoimmunotherapy. Blood. 2016; 128(3):395-404.

8. Rigolin GM, Cavallari M, Quaglia FM, et al. In CLL, comorbidities and the complex karyotype are associated with an inferior outcome independently of CLL-IPI. Blood. 2017;129(26):3495-2498.

9. Le Bris Y, Struski S, Guieze R, et al. Major prognostic value of complex karyotype in addition to TP53 and IGHV mutational status in first-line chronic lymphocytic leukemia. Hematol Oncol. 2017;35(4):664 670.

10. Rigolin GM, del Giudice I, Formigaro L, et al. Chromosome aberrations detected by conventional karyotyping using novel mitogens in chronic lymphocytic leukemia: Clinical and biologic correlations. Genes Chromosomes Cancer. 2015;54(12):818826.

11. Badoux XC, Keating MJ, Wang X, et al. Fludarabine, cyclophosphamide, and rituximab chemoimmunotherapy is highly effective treatment for relapsed patients with CLL. Blood. 2011;117(11):3016-3024.

12. Anderson MA, Tam C, Lew TE, et al. Clinicopathological features and outcomes of progression of CLL on the BCL2 inhibitor venetoclax. Blood. 2017; 129(25):3362-3370

13. Thompson PA, O'Brien SM, Wierda WG, et al. Complex karyotype is a stronger predictor than del(17p) for an inferior outcome in relapsed or refractory chronic lymphocytic leukemia patients treated with ibrutinibbased regimens. Cancer. 2015; 121(20): 3612-3621.

14. Baliakas $\mathrm{P}$, Jeromin $\mathrm{S}$, Iskas $\mathrm{M}$, $\mathrm{F}$, et al. Cytogenetic complexity in chronic lymphocytic leukemia: definitions, associations, and clinical impact. Blood. 2019; 133(11):1205-1216.

15. Rigolin GM, Saccenti E, Guardalben E, et al. In chronic lymphocytic leukaemia with complex karyotype, major structural abnormalities identify a subset of patients with inferior outcome and distinct biological characteristics. Br J Haematol. 2018; 181(2):229-233

16. Visentin A, Bonaldi L, Rigolin GM, et al. The combination of complex karyotype subtypes and IGHV mutational status identifies new prognostic and predictive groups in chronic lymphocytic leukaemia. Br J Cancer. 2019;121(2):150-156
17. Rigolin GM, Saccenti E, Melandri A, et al. In chronic lymphocytic leukaemia, SLAMF1 deregulation is associated with genomic complexity and independently predicts a worse outcome. Br J Haematol. 2021;192(6):1068-1072

18. Cavallari M, Cavazzini F, Bardi A, et al Biological significance and prognostic/predictive impact of complex karyotype in chronic lymphocytic leukemia. Oncotarget. 2018;9(76):34398-34412.

19. Hallek M, Cheson BD, Catovsky D, et al Guidelines for the diagnosis and treatment of chronic lymphocytic leukemia: a report from the International Workshop on Chronic Lymphocytic Leukemia updating the National Cancer Institute-Working Group 1996 guidelines. Blood. 2008; 111(12):5446-5456.

20. Hallek M. Chronic lymphocytic leukemia: 2015 Update on diagnosis, risk stratification, and treatment. Am J Hematol. 2015:90(5):446-460.

21. Langerak AW, Davi F, Ghia P, et al Immunoglobulin sequence analysis and prognostication in CLL: guidelines from the ERIC review board for reliable interpretation of problematic cases. Leukemia. 2011; 25(6):979-984.

22. Malcikova J, Tausch E, Rossi D, et al. ERIC recommendations for TP53 mutation analysis in chronic lymphocytic leukemiaupdate on methodological approaches and results interpretation. Leukemia. 2018 32(5):1070-1080.

23. Blanco G, Puiggros A, Baliakas P, et al Karyotypic complexity rather than chromosome 8 abnormalities aggravates the outcome of chronic lymphocytic leukemia patients with TP53 aberrations Oncotarget. 2016;7(49):80916-80924.

24. Kreinitz N, Polliack A, Tadmor T. Chronic lymphocytic leukemia is becoming more complex: how to define complex karyotype? Leuk Lymphoma. 2018;59(3):521522. 
25. Terrin L, Trentin L, Degan M, et al. Telomerase expression in B-cell chronic lymphocytic leukemia predicts survival and delineates subgroups of patients with the same igVH mutation status and different outcome. Leukemia. 2007;21(5):965-972.

26. Raponi S, Del Giudice I, Marinelli M, et al. Genetic landscape of ultra-stable chronic lymphocytic leukemia patients. Ann Oncol. 2018;29(4):966-972.

27. Brochet X, Lefranc MP, Giudicelli V. IMGT/V-QUEST: the highly customized and integrated system for IG and TR standardized V-J and V-D-J sequence analysis. Nucl Acids Res. 2008;36(Web Server issue):W503-508.

28. Hamblin TJ, Davis Z, Gardiner A, Oscier DG, Stevenson FK. Unmutated Ig V(H) genes are associated with a more aggressive form of chronic lymphocytic leukemia. Blood. 1999;94(6):1848-1854.

29. Visentin A, Facco M, Gurrieri C, et al. Prognostic and predictive effect of IGHV mutational status and load in chronic lymphocytic leukemia: focus on FCR and BR treatments. Clin Lymphoma Myeloma Leuk. 2019;19(10):678-685.

30. Bystry V, Agathangelidis A, Bikos V, Set al. ARResT/assignsubsets: a novel application for robust subclassification of chronic lymphocytic leukemia based on B cell receptor IG stereotypy. Bioinformatics. 2015; 31(23): 3844-3846.

31. Agathangelidis A, Chatzidimitriou A, Gemenetzi K, et al. Higher-order connections between stereotyped subsets: implications for improved patient classification in CLL. Blood. 2021;137(10):1365-1376.

32. Dohner H, Stilgenbauer S, Benner A, et al. Genomic aberrations and survival in chronic lymphocytic leukemia. N Engl J Med. 2000;343(26):1910-1916.

33. Rossi D, Rasi S, Fabbri G, et al. Mutations of NOTCH1 are an independent predictor of survival in chronic lymphocytic leukemia. Blood. 2012;119(2):521-529.

34. Al-Sawaf O, Robrecht S, Bahlo J, et al. Richter transformation in chronic lymphocytic leukemia (CLL)-a pooled analysis of German CLL Study Group (GCLLSG) front line treatment trials. Leukemia. 2021; 35(1): 169-176.

35. Harrell FE Jr, Lee KL, Mark DB. Multivariable prognostic models: issues in developing models, evaluating assumptions and adequacy, and measuring and reducing errors. Stat Med. 1996;15(4):361-387.

36. Iba K, Shinozaki T, Maruo K, Noma H. Reevaluation of the comparative effectiveness of bootstrap-based optimism correction methods in the development of multivariable clinical prediction models. BMC Med Res Methodol. 2021;21(1):9.

37. Cohen JA, Rossi FM, Zucchetto A, et al. A laboratory-based scoring system predicts early treatment in Rai 0 chronic lymphocytic leukemia. Haematologica. 2020; 105(6):1613-1620.

38. International CLLIPIwg. An international prognostic index for patients with chronic lymphocytic leukaemia (CLL-IPI): a metaanalysis of individual patient data. Lancet Oncol. 2016;17(6):779-790

39. Delgado J, Doubek M, Baumann T, et al. Chronic lymphocytic leukemia: a prognostic model comprising only two biomarkers (IGHV mutational status and FISH cytogenetics) separates patients with different outcome and simplifies the CLL-IPI. Am J Hematol. 2017;92(4):375-380.

40. Condoluci A, Rossi D. Richter syndrome. Curr Oncol Rep. 2021;23(3):26.

41. Ayers EC, Mato AR. Richter's Transformation in the era of kinase inhibitor therapy: a review. Clin Lymphoma Myeloma Leuk. 2017;17(1):1-6.

42. Visentin A, Imbergamo S, Scomazzon E, et al. BCR kinase inhibitors, idelalisib and ibrutinib, are active and effective in Richter syndrome. Br J Haematol. 2019;185(1):193197.

43. Ding W, LaPlant BR, Call TG, et al. Pembrolizumab in patients with CLL and Richter transformation or with relapsed CLL. Blood. 2017;129(26):3419-3427.

44. Rossi D, Spina V, Gaidano G. Biology and treatment of Richter syndrome. Blood. 2018;131(25):2761-2772.

45. Fabbri G, Khiabanian $\mathrm{H}$, Holmes $A B$, et al. Genetic lesions associated with chronic lymphocytic leukemia transformation to Richter syndrome. J Exp Med. 2013; 210(11):2273-2288

46. Miller CR, Ruppert AS, Heerema NA, et al. Near-tetraploidy is associated with Richter transformation in chronic lymphocytic leukemia patients receiving ibrutinib. Blood Adv. 2017;1(19):1584-1588.

47. Rogers KA, Huang Y, Ruppert AS, et al. A single-institution retrospective cohort study of first-line R-EPOCH chemoimmunotherapy for Richter syndrome demonstrating complex chronic lymphocytic leukaemia karyotype as an adverse prognostic factor. Br J Haematol. 2018; 180(2):259-266

48. Wang Y, Tschautscher MA, Rabe KG, et al. Clinical characteristics and outcomes of
Richter transformation: experience of 204 patients from a single center. Haematologica. 2020;105(3):765-773.

49. Baliakas P, Puiggros A, Xochelli A, et al Additional trisomies amongst patients with chronic lymphocytic leukemia carrying trisomy 12: the accompanying chromosome makes a difference. Haematologica. 2016; 101(7):e299-302.

50. Puiggros A, Collado R, Calasanz MJ, et al. Patients with chronic lymphocytic leukemia and complex karyotype show an adverse outcome even in absence of TP53/ATM FISH deletions. Oncotarget. 2017;8(33):54297-54303.

51. Ramos-Campoy S, Puiggros A, Bea S, et al. Chromosome banding analysis and genomic microarrays are both useful but not equivalent methods for genomic complexity risk stratification in chronic lymphocytic leukemia patients. Haematologica. 2022, 107(3):593-603.

52. Morabito F, Del Poeta G, Mauro FR, et al. TP53 disruption as a risk factor in the era of targeted therapies: a multicenter retrospective study of 525 chronic lymphocytic leukemia cases. Am J Hematol. 2021;96(8): E306-E310.

53. Woyach JA, Ruppert AS, Heerema NA, et al Ibrutinib regimens versus chemoimmunotherapy in older patients with untreated CLL. N Engl J Med. 2018;379(26):25172528.

54. Kreuzer KA, Furman RR, Stilgenbauer S, et al. The impact of complex karyotype on the overall survival of patients with relapsed chronic lymphocytic leukemia treated with idelalisib plus rituximab. Leukemia. 2020;34(1):296-300.

55. Rigolin GM, Cavazzini F, Piciocchi A, et al. Efficacy of idelalisib and rituximab in relapsed/refractory chronic lymphocytic leukemia treated outside of clinical trials. a report of the gimema working group. Hematol Oncol. 2021;39(3):326-335.

56. Visentin A, Frezzato F, Severin F, et al. Lights and shade of next-generation Pi3k inhibitors in chronic lymphocytic leukemia. Onco Targets Ther. 2020; 13:9679-9688.

57. Al-Sawaf O, Lilienweiss E, Bahlo J, et al. High efficacy of venetoclax plus obinutuzumab in patients with complex karyotype and chronic lymphocytic leukemia. Blood. 2020;135(11):866-870.

58. Jebaraj BMC, Tausch E, Landau DA, et al. Short telomeres are associated with inferior outcome, genomic complexity, and clonal evolution in chronic lymphocytic leukemia. Leukemia. 2019;33(9):2183-2194. 